\title{
Targeting of growth factors in the treatment of hepatocellular carcinoma: The potentials of polysaccharides (Review)
}

\author{
XUAN WANG $^{1}$, JIEYU DING ${ }^{1}$, YUANYUAN FENG $^{2}$, LINGLING WENG ${ }^{1}$, GUANGQIANG ZHAO ${ }^{1}$, \\ JIANFENG XIANG $^{1}$, MINGUANG ZHANG ${ }^{1}$ and DONGWEI XING ${ }^{1}$ \\ ${ }^{1}$ Radiology Department, Shanghai Municipal Hospital of Traditional Chinese Medicine Affiliated \\ to Shanghai University of Traditional Chinese Medicine, Shanghai 200071; ${ }^{2}$ Oncology Department, \\ Shuguang Hospital, Shanghai University of Traditional Chinese Medicine, Shanghai 200021, P.R. China
}

Received March 18, 2015; Accepted August 19, 2016

DOI: $10.3892 / \mathrm{ol} .2017 .5602$

\begin{abstract}
Hepatocellular carcinoma (HCC) has become a leading cause of cancer-associated mortality worldwide and is thus of great concern. Although various chemotherapeutic drugs are currently used for the treatment of HCC, severe side effects associated with these treatments have prompted interest in novel therapies, including the use of certain biological macromolecules such as polysaccharides. Several studies have shown that polysaccharides have anticancer and antiproliferative effects on HCC. Vascular endothelial growth factor, transforming growth factor $\beta$, epidermal growth factor and fibroblast growth factor may be effective targets for polysaccharides and may modulate tumor growth and immunity through increasing the expression levels of cytokines. The present review focuses on the ways in which growth factors contribute to the development of HCC, and on the anti-growth factor activities of natural and synthetic polysaccharides, as well as their effect on proinflammatory cytokines.
\end{abstract}

\section{Contents}

1. Introduction

2. Involvement of growth factors in HCC

3. Growth factor signaling as a therapeutic target in HCC: Potential of polysaccharides

4. Conclusion and future perspectives

Correspondence to: Professor Minguang Zhang or Dr Dongwei Xing, Radiology Department, Shanghai Municipal Hospital of Traditional Chinese Medicine Affiliated to Shanghai University of Traditional Chinese Medicine, 274 Zhijiang Road, Shanghai 200071, P.R. China

E-mail: mgzhang09@163.com

E-mail: szyxingdongwei@163.com

Key words: hepatocellular carcinoma, growth factors, polysaccharides

\section{Introduction}

Hepatocellular carcinoma (HCC) is the second leading cause of cancer-associated mortality worldwide (1). HCC represents the most common histological type of liver cancer, accounting for $70-85 \%$ of cases (2). Its treatment may include local ablation, surgical resection, trans-catheter arterial chemoembolization and the administration of chemotherapeutic agents $(3,4)$. However, at the time of their diagnosis, HCC tumors may be too large or may have expanded into nearby major blood vessels or metastasized, rendering the majority of HCC patients unsuitable for treatment by surgical resection (5). In addition, chemotherapy provides only a modest improvement to the overall survival time of patients due to the lack of specificity of the agent as well as the intolerable side effects that are often induced (6). Thus, novel anticancer therapeutic agents for use in the treatment of HCC are urgently required. In this regard, polysaccharides may be promising candidates. Numerous studies have reported that polysaccharides have antitumor activities and that polysaccharides from different herbs have different roles (7-10).

Normal cells in multicellular organisms constantly signal to one another via molecules called growth factors and cytokines. The signals conducted by growth factors and cytokines can inform individual cells whether to divide or not (11). According to Hanahan and Weinberg (12), the cell surface receptors that transduce signals into the cell are targets that can lead to dysregulation during tumor progression, resulting in self-sufficiency in growth signaling, one of the major hallmarks of cancer cells. Growth factor receptors are overexpressed in numerous types of cancer, and may enable the cancer cells to become hyper-responsive to ambient levels of growth factors and even ligand-independent signaling (13). This observation provides the rationale for research into the development of anti-growth factor compounds. The present study reviews the mechanisms that underlie the growth factor-mediated growth, proliferation, angiogenesis and metastasis of HCC cells, how they may be targeted by polysaccharides, and the current research being conducted into the use of these polysaccharides for the treatment of HCC. 


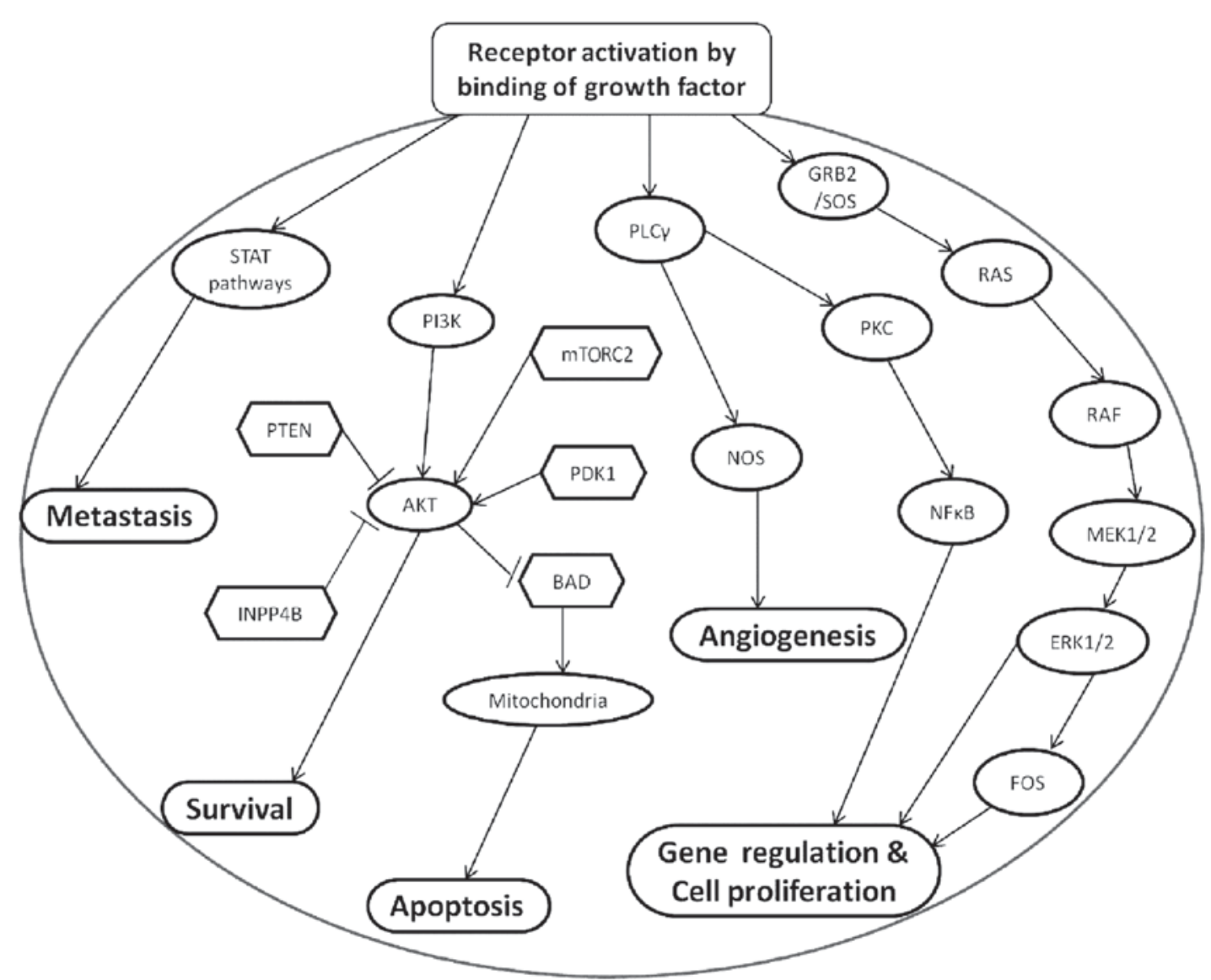

Figure 1. Tumorigenesis, mediated by growth factors. Growth factors activates their corresponding receptors, leading to the subsequent activation of four pathways: PI3K/AKT/mTOR pathway, Ras/Raf/MAPK pathway, NOS pathway and STAT pathway. These four pathways can induce cell proliferation, survival, angiogenesis and metastasis. PI3K, phosphoinositide 3-kinase; AKT, protein kinase B; mTOR, mammalian target of rapamycin; MAPK, mitogen-activated protein kinase; NOS, nitric oxide synthase; STAT, Signal Transducer and Activator of Transcription; GRB2/SOS, growth factor receptor-bound protein 2/Son of Sevenless; PLC $\gamma$, phospholipase C $\gamma$; PKC, protein kinase C; PTEN, phosphatase and tensin homolog; mTORC2, mTOR complex 2; PDK1, pyruvate dehydrogenase kinase 1; BAD, Bcl2-associated agonist of cell death; INPP4B, inositol polyphosphate-4-phosphatase type II B; NFkB, nuclear factor кB; MEK1/2, MAPK kinase 1/2; ERK1/2, extracellular signal-regulated kinase 1/2.

\section{Involvement of growth factors in HCC}

Upon response to their specific ligands, growth factor receptors mediate tumorigenic activity through a variety of signaling pathways (14). A large number of growth factors are produced in the human liver during the foetal stage, including epidermal growth factor (EGF), insulin-like growth factors (IGFs), hepatocyte growth factor (HGF), vascular endothelial growth factor (VEGF), fibroblast growth factor (FGF), platelet-derived growth factor (PDGF), transforming growth factor (TGF)- $\alpha$ and TGF- $\beta$. However, the production of many of these growth factors decreases or is absent in the normal adult liver (15-17). On the other hand, following injury or damage, when liver regeneration is required, adult hepatocytes are able to upregulate the production of particular growth factors, including EGF, TGF- $\alpha$, IGF and VEGF (18). This normal, transient upregulation is dysregulated in the chronically injured liver, and such dysregulation of growth factor production and growth factor receptor signaling in adult hepatocytes serves an important role in hepatocarcinogenesis (19) (Fig. 1).

VEGFs, prominent factors associated with aggressive tumor behavior, belong to the PDGF supergene family $(20,21)$. VEGF-A, the major factor for angiogenesis, binds to two tyrosine kinase receptors, namely VEGF receptor (VEGFR)-1 and VEGFR-2. VEGFR-1 is a trans-membrane receptor and is responsible for mediating angiogenesis and inflammatory responses. VEGFR-2 serves important roles in physiological and pathological angiogenesis and regulates the proliferation and migration of endothelial cells $(22,23)$. Previous studies have indicated that high serum levels of VEGF have significant predictive ability for the estimation of survival in HCC patients treated by hepatic resection, radiofrequency ablation or trans-catheter arterial chemoembolization $(24,25)$. In HCC, VEGF expression is increased through the expression of hypoxia-inducible factor- $1 \alpha$. In hypoxic conditions, VEGF and VEGFR trigger the angiogenic cascade and promote endothelial cell migration and proliferation. Thus, VEGF blockade may be an effective target for HCC treatment $(26,27)$. Curcumin and Bevacizumab (VEGF blocker) can independently inhibit $\mathrm{HCC}$ progression through the regulation of the VEGF/VEGFR/K-ras pathway (28).

TGF- $\beta 1$ is commonly recognized as a hallmark of HCC and represents one of the most important pathways to be targeted (29). TGF- $\beta$ induces the epithelial-mesenchymal transition (EMT), which is involved in hepatocarcinogenesis and HCC metastasis (30). Stimulation of TGF- $\beta 1$ leads to the activation of phosphoinositide 3-kinase (PI3K)/protein 
kinase B (Akt), whereas inhibition of PI3K/Akt activation represses EMT and invasion of HCC cells (31).

EGF interacts with the EGF receptor (EGFR) to stimulate cell growth, proliferation and differentiation, and EGFR overexpression, which is known to be associated with tumorigenesis, occurs in $40-70 \%$ of cases of human HCC (32). In addition, FGF and HGF, which are heparin-binding growth factors, act as a potent mitogens for HCC (33). In summary, all of the aforementioned factors are associated with tumor growth and development, and the control of the expression of these growth factors can serve an important role in producing antitumor effects.

\section{Growth factor signaling as a therapeutic target in HCC: Potential of polysaccharides}

Clinical trials indicate that growth factor receptors and their associated signaling pathways are important in HCC cancer etiology and progression (34). Hence, growth factors, their receptors, and signaling pathways mediated by these growth factors are interesting targets for future therapeutic approaches. A number of strategies, including inhibition of receptor expression using gene therapy (antisense approach), antagonistic monoclonal antibodies that prevent the binding of ligands to receptors, or pharmacological (low-molecular-weight) receptor-selective tyrosine kinase inhibitors have already been evaluated for their potency in inhibiting the activity and downstream signaling cascades of these receptors in HCC (34).

Initial clinical trials have also demonstrated that multi-kinase inhibition is an effective novel treatment strategy in HCC (35). In this respect, sorafenib, an inhibitor of Raf, VEGF and PDGF signaling, is the first multi-kinase inhibitor that has been approved by the Food \& Drug Administration for the treatment of advanced HCC (35). Sorafenib administration is an effective treatment for advanced HCC and can increase the survival rate of these patients. Sorafenib inhibits the growth of hepatoma cells by interfering with the secretion of IGF-1 (36). In addition, linifanib, a multi-targeted receptor tyrosine kinase inhibitor, can inhibit members of the VEGF and PDGF receptor families (37).

However these drugs have their own disadvantages with regard to side effects, drug intolerance and resistance. Although sorafenib has shown promising therapeutic effects, primary and acquired resistance to the drug has been reported in numerous studies $(38,39)$. Furthermore, the overall survival time of HCC patients who responded to sorafenib improved by only $\sim 2$ months $(40,41)$. In this setting, polysaccharides may be a good option. Many polysaccharides and polysaccharide-protein complexes have been isolated from mushrooms, fungi, yeast, algae and plants. Polysaccharides have a broad spectrum of biological effects, such as antibiotic, antioxidant, anti-mutant, anticoagulant, immunomodulating and anticancer activities (42-48). The antitumor ability depends on a number of properties, such as structure, dose, mechanism of action, and site of activity (49). Several studies have demonstrated that polysaccharides may have roles in the prevention of tumorigenesis and induction of tumor cell apoptosis, immunomodulation during chemotherapy, and inhibition of tumor metastasis (50). Further research into how polysaccharides may act via growth factors, cytokine networks and signaling pathways, and their roles and mechanisms in cancer regulation is required.

Sources of polysaccharides targeting growth factors in HCC. Polysaccharides are the most abundant group of biopolymers and, due to their biocompatibility, biodegradability and non-toxicity, many studies have been conducted to evaluate their therapeutic effects (51-53). A large variety of polysaccharides can be isolated from plants (dietary fibers, herbs and wood plants), algae, lichen, microorganisms (fungi, yeasts and bacteria) and animals. Research has been conducted to characterize the constituents of these polysaccharides; however these kinds of studies are strikingly limited.

A study by Lv et al (54) found that polysaccharides isolated from tea were hetero-polysaccharides which consisted of mannose, ribose, rhamnose, glucuronic acid, galacturonic acid, glucose, xylose, galactose and arabinose with molar contents of 16.3, 10.3, 47.1, 5.6, 24.0, 128.4, 25.0, 101.4 and $71.1 \mu \mathrm{M}$, respectively (54). Another study demonstrating the hepatoprotective effect of polysaccharides from Huangshan Maofeng green tea also found that the main monosaccharide components of this polysaccharide were galactose (mol.\%, $35.0 \%)$, arabinose (28.9\%) and galacturonic acid (11.3\%) (55). Capillary zone electrophoresis analysis showed that polysaccharide isolated from Gynostemma pentaphyllum Makino consisted of glucose $(23.2 \%)$, galactose (18.9\%), arabinose $(10.5 \%)$, rhamnose $(7.7 \%)$, galacturonic acid $(4.7 \%)$, xylose (3.9\%), mannose (3.1\%), and glucuronic acid (1.2\%) (56). Characterization of polysaccharides from two Pleurotus mushrooms, $P$. eryngii and $P$. ostreatus, found that they were mainly composed of mannose, along with other monosaccharides, including glucose, galactose, xylose and rhamnose $(57,58)$. The monosaccharide constituents of Lentinus edodes polysaccharide have also been characterized (59).

However, although many studies have found that, grossly, these polysaccharides have potent antitumor activities in HCC, it has not been determined which components of the polysaccharides are responsible for such effects. Furthermore, synthetic polysaccharides are often used in association with chemotherapy (60-62). Traditional Chinese medicine (TCM) and certain herbal medicines have been used in the treatment of cancer for thousands of years in China, Japan and South Korea, as well as some other Asian countries (63). As adjunct anticancer agents, TCMs can have important anticancer roles in inducing apoptosis and differentiation, improving the immune system, inhibiting angiogenesis, and also in increasing the sensitivity to and reducing the side effects of chemotherapeutics, and improving patient survival time (64). For example, naturopathic therapy using Cordyceps sinensis can prolong the survival time of patients with HCC (65). Studies have demonstrated that their roles in HCC are associated with the regulation of growth factors and cytokines. The names, sources and specific targets of polysaccharides are given in Table I.

Polysaccharides targeting a single growth factor. Pleurotus mushroom polysaccharide-protein complex (PP) has shown anticancer activities against liver cancer cells in vitro and 
Table I. Names of polysaccharides with their sources and potential targets.

A, Polysaccharides from natural sources

Source and name

Targets

Traditional Chinese medicine

Aconitum koreanum polysaccharides

Salvia miltiorrhiza polysaccharides

Radix Glycyrrhizae polysaccharide

Astragalus polysaccharides

Exopolysaccharide fraction from Cordyceps sinensis

Plant-derived

Tea carbohydrate polymers

Dihydromyricetin

Corn silk polysaccharides

Gynostemma pentaphyllum Makino polysaccharide

Artemisia apiacea polysaccharide

Dietary

Pleurotus mushroom polysaccharide

Tricholoma matsutake polysaccharide

Lentinus edodes polysaccharide

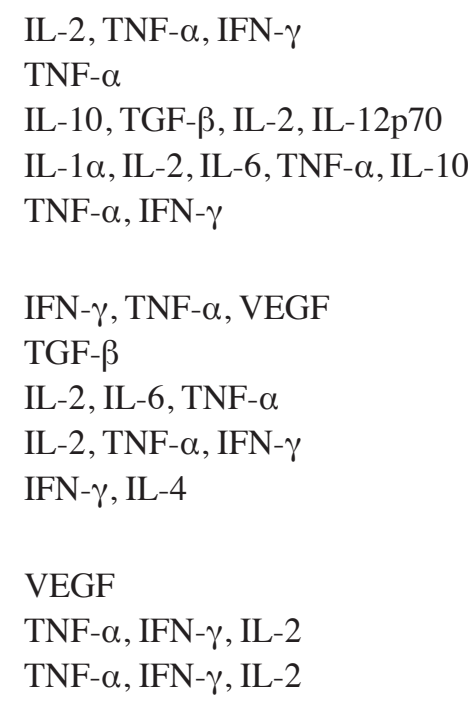

B, Synthetic polysaccharides

Name

Targets

Low molecular weight chitosan

Chitosan nanoparticles

Galactose modified trimethyl chitosan-cysteine nanoparticles

VEGF

VEGFR-2

$\mathrm{VEGF}$

C, Others

Name

Targets

HS mimetic PI-88 series

FGF1, FGF2

HS mimetic PG500 series

FGF1, FGF2, VEGF

HS, heparan sulfate; IL, interleukin; TNF, tumor necrosis factor; IFN- $\gamma$, interferon- $\gamma$; FGF, fibroblast growth factor; TGF- $\beta$, transforming growth factor- $\beta$; VEGF, vascular endothelial growth factor; VEGFR-2, VEGF receptor 2.

in vivo (66). PP inhibits proliferation by inactivation of PI3K/Akt signaling (67). Studies have suggested that, in HCC, $\mathrm{PP}$ reduces the expression of secretory VEGF, which in turn mediates autocrine regulation of PI3K/Akt signaling in xenograft BALB/c nude mice. PP can also enhance sensitivity to the chemotherapeutic drug cisplatin (68).

Tea carbohydrate polymers are natural polymers with antioxidant, hepatoprotective and antitumor activities (69). These have exhibited strong antitumor activity in experimental HCC animals. It was found that administration of tea carbohydrate for 40 days could significantly inhibit tumour growth and that three different doses of carbohydrate treatment $(100,200$ and $300 \mathrm{mg} / \mathrm{kg}$ body weight/day) significantly decreased microvessel density in a dose-dependent manner. During this period, a significant enhancement in the serum white blood cell count and interferon (IFN) $-\gamma$ and tumor necrosis factor $\alpha(\mathrm{TNF}-\alpha)$ levels, and a decrease in the expression of VEGF and proliferating cell nuclear antigen were found in H22 tumor tissues (70). Tea carbohydrates were also found to augment the antitumor activity of doxorubicin (71).

Radix Glycyrrhizae polysaccharide (GP) is a major active compound extracted from Radix Glycyrrhizae, a commonly used traditional herbal medicine. In a study of tumor-bearing mice, the effect of GP on tumor growth inhibition was determined to be likely caused by the to upregulation of the Th1/Th2 cytokine ratio in serum by the decrease in the transcription factor Foxp3, interleukin (IL)-10 and transforming growth factor (TGF)- $\beta$ levels $(\mathrm{P}<0.01)$, and the increase in IL-2 and IL-12p70 levels in serum $(\mathrm{P}<0.01)(72)$. 
Dihydromyricetin (DHM) is the active component in extracts of Ampelopsis grossedentata. The principal anticancer mechanism of DHM is via the induction of p53-dependent apoptosis (73-75). A previous study demonstrated that DHM enhances chemosensitivity to nedaplatin (a platinum-containing chemotherapeutic drug) by activating the p53/B-cell lymphoma 2 (Bcl-2) signaling pathway, which resulted in mitochondrial dysfunction and induced cell death and growth inhibition in HCC cells (76). Furthermore, it has also been reported that induction of cell apoptosis by DHM is regulated via the TGF- $\beta / \mathrm{Smad} 3$ signaling pathway and that TGF- $\beta$ expression is decreased in the mouse HCC cell line Hepal-6 (77).

Polysaccharides targeting multiple growth factors. Cell surface/extracellular matrix heparan sulfate (HS) glycosaminoglycans are complex polysaccharides, and HS mimetics function by blocking these interactions and inhibiting processes crucial to tumor progression, and could therefore be considered as a novel class of cancer therapeutics $(78,79)$. The HS mimetic PI-88 acts as an inhibitor of heparanase and angiogenesis in HCC. A series of PI- 88 analogs with augmented chemical and biological properties are composed of single, defined oligosaccharides with specific modifications and can inhibit heparanase and FGF1, FGF2 and VEGF, and thus can act as potent inhibitors of growth factor-induced endothelial cell proliferation (80). The PG500 series of HS mimetics have high affinity for growth factors; however, their affinity is lower compared with the PI-88 series. A previous study showed that PG500 compounds containing a larger lipophilic moiety had reduced anticoagulant activity compared with PI-88 (81). However, the selected PG500 series compounds all inhibited FGF1, FGF2 and VEGF-induced endothelial cell proliferation. In a tube formation assay, it was found that certain of the PG500 series compounds (PG536, PG537, PG545 and PG562) inhibited tube formation by $>90 \%$ at a concentration of $10 \mu \mathrm{M}$. Furthermore, in a rat aortic assay of angiogenesis, it was found that PG536, PG545 and PG546 inhibited angiogenesis by $>80 \%$ following daily administration of $10 \mu \mathrm{M}$ for 6-8 days. Treatment with PG545, PG546 and PG547 also potently inhibited the development of metastatic nodules when administered at $10 \mathrm{mg} / \mathrm{kg}$. Finally, PG545 was found to significantly inhibit tumor development in a xenograft model (HT29 colon adenocarcinoma cells) (81).

Polysaccharides targeting pro-inflammatory cytokines. Cytokines are a subtype of growth factors that are produced by hematopoietic and immune cell types, and include IFNs and ILs. Cytokines normally function to stimulate a host response to control cellular stress and minimize cellular damage. Cytokines produced by CD4+ T-helper (Th) cells are categorized as Th1 or Th2; Th1 cytokines (e.g., IL-1 $\alpha$, IL-1 $\beta$, IL-2, IL-12p35, IL-12p40, IL-15, TNF- $\alpha$ and IFN- $\gamma$ ) and Th2 cytokines (e.g., IL-4, IL-8, IL-10, and IL-5) are generally referred to as proinflammatory and anti-inflammatory cytokines, respectively (82). In the liver, cell damage and regeneration mediated by viral hepatitis-induced immune responses can cause dysregulated hepatocyte proliferation, which may accelerate the development and progression of hepatic cancer through transcription and activation of cytokines and growth factors, oxidative DNA damage, DNA methylation, and hepatocyte injury (83-86). Increasing evidence indicates the involvement of cytokines in hepatocarcinogenesis [reviewed by Bishayee (87)].

On the other hand, numerous studies have demonstrated that cytokines have broad antitumor activity. Accordingly, proinflammatory cytokines, including TNF- $\alpha$, IL- 6 and IL-1 $\beta$, and transcription factors that are required for signaling by these cytokines, including NF- $\mathrm{KB}$ and STATs, are emerging as potential targets for anticancer therapy (88). Serum cytokines play important roles in suppressing tumor growth (89). IL- $1 \alpha$, IL-2 and IL- 6 are capable of inducing the proliferation of responsive T-cells (90). Wnt/ $\beta$-catenin and EGFR signaling are the major cascades that are activated through proinflammatory cytokines and involved in the progression of epithelial tumors (91). IL-10 inhibits the synthesis of IL-2 and TNF- $\alpha$ (92).

Several in vivo experiments have demonstrated that polysaccharides from various sources have the ability to modulate tumor growth and immunity. Most affect tumor growth and immune functions in hepatocarcinoma tumor-bearing mice by increasing the expression levels of IL-2, IL-6 and TNF- $\alpha$, and can inhibit the growth of hepatoma and prolong the survival time of these mice. These polysaccharides include those from Corn silk (Zea mays L.) (93), Aconitum koreanum and Salvia miltiorrhiza (94).

Polysaccharides from Astragalus membranaceus (APS) have been widely studied for their anticancer potential. APS was found to exert antitumor effects in H22 tumor-bearing mice and also enhanced chemosensitivity to adriamycin (ADM). The antitumor and synergistic activity of APS may be associated with its effect of increasing the expression levels of IL-1 $\alpha$, IL-2, IL-6 and TNF- $\alpha$ and decreasing IL-10 levels. APS was also found to downregulate multidrug resistance 1 mRNA and P-glycoprotein expression levels, which may be related to other anticancer effects $(10,95)$. Several studies aimed to assess the antitumor potentials of polysaccharides from different sources and found that their anticancer effects are mediated by the secretion of INF- $\gamma$. These included polysaccharides obtained from G. pentaphyllum Makino (96), a polysaccharide isolated from Artemisia apiacea (97), and oral administration of A. coreanum (98). Polysaccharides obtained from cultured Cordyceps fungus have also been shown to have pharmacological efficacy; specifically, the exopolysaccharide fraction from Cordyceps sinensis was found to significantly inhibit HCC tumor growth and lead to elevated TNF- $\alpha$ and IFN- $\gamma$ mRNA expression of splenic lymphocytes (99). Collectively, these data indicate the increasing contribution to therapeutic effects of these polysaccharides.

Combination of polysaccharides. 5-fluorouracil (5-FU) is a chemotherapeutic drug that is commonly used in HCC treatment. Chemotherapeutic drugs combined with polysaccharide may act more effectively together; thus, a combination of polysaccharides can be used with chemotherapeutic drugs like 5-FU to enhance $\mathrm{H} 22$ cell growth inhibition. A previous study found that polysaccharides from Tricholoma matsutake (PTM) could activate splenic lymphocytes, and another study demonstrated that polysaccharides from Lentinus edodes (PL) had antitumor bioactivities at concentrations ranging from 
50 to $500 \mu \mathrm{g} / \mathrm{ml}$, with maximum inhibition occurring at a concentration of $200 \mu \mathrm{g} / \mathrm{ml}$ between 36 and $48 \mathrm{~h}(100,101)$. Notably, in vivo experiments revealed significant increases in the cytotoxic $\mathrm{T}$ lymphocyte and natural killer cell activities, the frequencies of CD4+ and CD8+ T cells in the spleen, and the serum levels of TNF- $\alpha$, IL- 2 and IFN- $\gamma$ in mice treated with 5-FU+PTM+PL when compared with mice treated with 5-FU, PTM or PL alone, 5-FU+PL, or 5-FU+PTM (102).

Helper polysaccharides. Polysaccharides have been studied for their roles in facilitating successful drug delivery, and it was demonstrated that certain polysaccharides are important in this regard.

In certain experimental tumor models, VEGF silencing by RNA interference (RNAi) has been attempted and has achieved positive results $(103,104)$; however, due to the poor stability of small interfering RNA (siRNA) molecules in vivo and the low cellular uptake, a safe and efficient carrier is required for therapeutic RNAi applications (105). Chitosan is a natural polysaccharide composed of randomly distributed $\beta$-[1-4]-linked D-glucosamine and N-acetyl-D-glucosamine. Low molecular weight chitosan (LMWC) can be used as a carrier for RNAi drugs directed against VEGF (106). It was found that LMWC/VEGF shRNA complexes could be efficiently transfected into murine hepatocarcinoma Hepa1-6 cells and that this could inhibit VEGF expression and suppress tumor angiogenesis (106). In another study, chitosan nanoparticles (CNP) were shown to significantly inhibit tumor growth and induce tumor necrosis in model mice xenografted with HCC (BEL-7402) cells. The dose-dependent tumor suppression by CNP was associated with the inhibition of tumor angiogenesis. Further mechanistic evidence suggested that this inhibition of tumor angiogenesis was linked to altered levels of VEGFR-2 (107).

In another study, tumor-bearing mice were orally administered with VEGF siRNA and pDNA expressing shRNA specific for survivin (a member of the inhibitors of apoptosis family) loaded onto galactose-modified trimethyl chitosan-cysteine nanoparticles with various degrees of galactosylation. The loaded nanoparticles could effectively accumulate in the tumor tissues, resulting in the downregulation of antiapoptotic survivin, which acted synergistically with the siRNA-mediated inhibition of angiogenic VEGF. This ultimately led to increased apoptosis and inhibition of angiogenesis in hepatoma (108).

\section{Conclusion and future perspectives}

To date, a number of standard therapies have been shown to result in modest improvements to the overall survival and quality of life of HCC patients. However, they are associated with significant toxicities. Furthermore, the ability of solid tumors to develop multiple invasion and resistance pathways that allow them to circumvent inhibition by a single signaling pathway is becoming increasingly evident (109). Expanding knowledge of the molecular signaling that underlies tumor cell resistance and the poor survival of patients with $\mathrm{HCC}$ can facilitate the development of new drugs with better safety profiles. Furthermore, resistance is less likely to arise in response to natural compounds (110).
As reviewed by Zong et al, the anticancer efficacy of polysaccharides was first recognized in 1946 (44). Since then numerous studies have suggested that polysaccharides can be utilized as key ingredients for bio-based materials in life sciences, including pharmaceuticals, and many polysaccharides have demonstrated potential as anticancer agents (96-99). With regard to drawbacks, such as low safety profiles and resistance of current therapies, the development of polysaccharides as treatment strategies for HCC is necessary.

Although there is potential for the use of these kinds of polysaccharides against HCC, research into these polysaccharides is limited. Regarding self-sufficiency in growth signaling, a major hallmark of cancer, polysaccharides have been shown to have good activity; as discussed in the present study, polysaccharides from different sources have shown moderate anticancer activity in $\mathrm{HCC}$ by targeting growth factors. However, an active area of research should be dedicated to the development of more efficient and economic approaches for the preparation and modification of polysaccharides, and to elucidating the structure-activity association. The active component of these polysaccharides must be identified to better understand their anticancer mechanism in HCC, and it is also important to pay attention to the signaling pathways that are modified by the use of polysaccharides. Furthermore it is essential that in vivo studies are performed to confirm the results obtained in vitro, and these polysaccharides must also be tested in a clinical setting.

Considering the limited therapeutic options available to treat HCC, studies investigating polysaccharides may provide a rationale for the translation of these compounds into potential therapeutics against HCC. Overall, recent discussion is indicating a bright future for polysaccharides targeting growth factors in $\mathrm{HCC}$.

\section{Acknowledgements}

This study was funded by the Shanghai Natural Science Foundation of China (grant no. 15ZR1438900), Shanghai Construction Doctoral Research (grant no. B201404) and Shanghai Municipal Health Bureau Scientific Research Program (grant no. 20134167).

\section{References}

1. Jemal A, Siegel R, Xu J and Ward E: Cancer statistics, 2010. CA Cancer J Clin 60: 277-300, 2010.

2. AC Society: Global cancer facts and figures 2008 http://www.cancer.org/acs/groups/content/@ editorial/documents/document/acspc-044552.pdf. Accessed: December 16, 2016.

3. Lencioni R, Petruzzi P and Crocetti L: Chemoembolization of hepatocellular carcinoma. Semin Intervent Radiol 30: 3-11, 2013.

4. Kim HY and Park JW: Clinical trials of combined molecular targeted therapy and locoregional therapy in hepatocellular carcinoma: Past, present and future. Liver Cancer 3: 9-17, 2014.

5. Levin B and Amos C: Therapy of unresectable hepatocellular carcinoma. N Engl J Med 332: 1294-1296, 1995.

6. Nocentini G: Ribonucleotide reductase inhibitors: New strategies for cancer chemotherapy. Crit Rev Oncol Hematol 22: 89-126, 1996.

7. Deng C, Fu H, Teng L, Hu Z, Xu X, Chen J and Ren T: Anti-tumor activity of the regenerated triple-helical polysaccharide from Dictyophora indusiata. Int J Biol Macromol 61: 453-458, 2013 
8. Shen H, Tang G, Zeng G, Yang Y, Cai X, Li D, Liu H and Zhou N: Purification and characterization of an antitumor polysaccharide from Portulaca oleracea L. Carbohydr Polym 93: 395-400, 2013

9. Yang Z, Xu J, Fu Q, Fu X, Shu T, Bi Y and Song B: Antitumor activity of a polysaccharide from Pleurotus eryngii on mice bearing renal cancer. Carbohydr Polym 95: 615-620, 2013.

10. Yang B, Xiao B and Sun T: Antitumor and immunomodulatory activity of Astragalus membranaceus polysaccharides in $\mathrm{H} 22$ tumor-bearing mice. Int J Biol Macromol 62: 287-290, 2013.

11. Odenthal J, Takes R and Friedl P: Plasticity of tumor cell invasion: Governance by growth factors and cytokines. Carcinogenesis 37: 1117-1128, 2016.

12. Hanahan D and Weinberg RA: The hallmarks of cancer Cell 100: 57-70, 2000.

13. Hanada K, Perry-Lalley DM, Ohnmacht GA, Bettinotti MP and Yang JC: Identification of fibroblast growth factor-5 as an overexpressed antigen in multiple human adenocarcinomas. Cancer Res 61: 5511-5516, 2001.

14. Habib SA, Aggour YA and Taha HA: Downregulation of transforming growth factor- $\beta$ (TGF-) and vascular endothelial growth factor (VEGF) in ehrlich ascites carcinoma-bearing mice using stearic acid-grafted carboxymethyl chitosan (SA-CMC). Nat Sci 4: 808-818, 2012

15. Wolf HK, Zarnegar R and Michalopoulos GK: Localization of hepatocyte growth factor in human and rat tissues: An immunohistochemical study. Hepatology 14: 488-494, 1991.

16. Awuah PK, Nejak-Bowen KN and Monga SP: Role and regulation of PDGFR $\alpha$ signaling in liver development and regeneration. Am J Pathol 182: 1648-1658, 2013.

17. Suzuki A, Iwama A, Miyashita $\mathrm{H}$, Nakauchi $\mathrm{H}$ and Taniguchi $\mathrm{H}$ Role for growth factors and extracellular matrix in controlling differentiation of prospectively isolated hepatic stem cells Development 130: 2513-2524, 2003.

18. Duncan SA: Mechanisms controlling early development of the liver. Mech Dev 120: 19-33, 2003

19. Breuhahn K, Longerich T and Schirmacher P: Dysregulation of growth factor signaling in human hepatocellular carcinoma. Oncogene 25: 3787-3800, 2006.

20. Shibuya M: Vascular endothelial growth factor and its receptor system: Physiological functions in angiogenesis and pathological roles in various diseases. J Biochem 153: 13-39, 2013.

21. Shibuya M and Claesson-Welsh L: Signal transduction by VEGF receptors in regulation of angiogenesis and lymphangiogenesis. Exp Cell Res 312: 549-560, 2006

22. Bhardwaj S, Roy H, Babu M, Shibuya M and Yla-Herttuala S: Adventitial gene transfer of VEGFR-2 specific VEGF-E chimera induces MCP-1 expression in vascular smooth muscle cells and enhances neointimal formation. Atherosclerosis 219 : 84-91, 2011

23. Meyer JP, Edwards KJ, Kozlowski P, Backer MV, Backer JM and Lewis JS: Selective imaging of VEGFR-1 and VEGFR-2 using 89Zr-labeled single-chain VEGF mutants. J Nucl Med 57 1811-1816, 2016

24. Kanematsu M, Semelka RC, Osada S and Amaoka N: Magnetic resonance imaging and expression of vascular endothelial growth factor in hepatocellular nodules in cirrhosis and hepatocellular carcinomas. Top Magn Reson Imaging 16: 67-75, 2005.

25. Schoenleber SJ, Kurtz DM, Talwalkar JA, Roberts LR and Gores GJ: Prognostic role of vascular endothelial growth factor in hepatocellular carcinoma: Systematic review and meta-analysis. Br J Cancer 100: 1385-1392, 2009

26. Raskopf E, Dzienisowicz C, Hilbert T, Rabe C, Leifeld L, Wernert N, Sauerbruch T, Prieto J, Qian C, Caselmann WH and Schmitz V: Effective angiostatic treatment in a murine metastatic and orthotopic hepatoma model. Hepatology 41: 1233-1240, 2005.

27. Tan HY, Wang N, Tsao SW, Zhang Z and Feng Y: Suppression of vascular endothelial growth factor via inactivation of eukaryotic elongation factor 2 by alkaloids in Coptidis rhizoma in hepatocellular carcinoma. Integr Cancer Ther 13: 425-434, 2014.

28. Gao JZ, DU JL, Wang YL, Li J, Wei LX and Guo MZ: Synergistic effects of curcumin and bevacizumab on cell signaling pathways in hepatocellular carcinoma. Oncol Lett 9: 295-299, 2015

29. Hoshida Y, Nijman SM, Kobayashi M, Chan JA, Brunet JP, Chiang DY, Villanueva A, Newell P, Ikeda K, Hashimoto M, et al: Integrative transcriptome analysis reveals common molecular subclasses of human hepatocellular carcinoma. Cancer Res 69: 7385-7392, 2009.
30. Ru NY, Wu J, Chen ZN and Bian H: HAb18G/CD147 is involved in TGF- $\beta$-induced epithelial-mesenchymal transition and hepatocellular carcinoma invasion. Cell Biol Int 39: 44-51, 2015.

31. Yu W, Huang C, Wang Q, Huang T, Ding Y, Ma C, Ma H and Chen W: MEF2 transcription factors promotes EMT and invasiveness of hepatocellular carcinoma through TGF- $\beta 1$ autoregulation circuitry. Tumour Biol 35: 10943-10951, 2014.

32. Buckley AF, Burgart LJ, Sahai V and Kakar S: Epidermal growth factor receptor expression and gene copy number in conventional hepatocellular carcinoma. Am J Clin Pathol 129: 245-251, 2008.

33. Lai JP, Chien JR, Moser DR, Staub JK, Aderca I, Montoya DP, Matthews TA, Nagorney DM, Cunningham JM, Smith DI, et al: hSulf1 Sulfatase promotes apoptosis of hepatocellular cancer cells by decreasing heparin-binding growth factor signaling. Gastroenterology 126: 231-248, 2004.

34. Höpfner M, Schuppan D and Scherübl H: Growth factor receptors and related signalling pathways as targets for novel treatment strategies of hepatocellular cancer. World J Gastroenterol 14 $1-14,2008$.

35. 35. Wilhelm SM, Adnane L, Newell P, Villanueva A, Llovet JM and Lynch M: Preclinical overview of sorafenib, a multikinase inhibitor that targets both Raf and VEGF and PDGF receptor tyrosine kinase signaling. Mol Cancer Ther 7: 3129-3140, 2008

36. Sprinzl MF, Puschnik A, Schlitter AM, Schad A, Ackermann K, Esposito I, Lang H, Galle PR, Weinmann A, Heikenwälder M and Protzer U: Sorafenib inhibits macrophage-induced growth of hepatoma cells by interference with insulin-like growth factor-1 secretion. J Hepatol 62: 863-870, 2015.

37. Albert DH, Tapang P, Magoc TJ, Pease LJ, Reuter DR, Wei RQ, Li J, Guo J, Bousquet PF, Ghoreishi-Haack NS, et al: Preclinical activity of ABT-869, a multitargeted receptor tyrosine kinase inhibitor. Mol Cancer Ther 5: 995-1006, 2006.

38. Blivet-Van Eggelpoël MJ, Chettouh H, Fartoux L, Aoudjehane L, Barbu V, Rey C, Priam S, Housset C, Rosmorduc O and Desbois-Mouthon C: Epidermal growth factor receptor and HER-3 restrict cell response to sorafenib in hepatocellular carcinoma cells. J Hepatol 57: 108-115, 2012.

39. Zhai B and Sun XY: Mechanisms of resistance to sorafenib and the corresponding strategies in hepatocellular carcinoma. World J Hepatol 5: 345-352, 2013

40. Bruix J and Sherman M; American Association for the Study of Liver Diseases: Management of hepatocellular carcinoma: An update. Hepatology 53: 1020-1022, 2011.

41. Cheng AL, Kang YK, Chen Z, Tsao CJ, Qin S, Kim JS, Luo R, Feng J, Ye S, Yang TS, et al: Efficacy and safety of sorafenib in patients in the Asia-Pacific region with advanced hepatocellular carcinoma: A phase III randomised, double-blind, placebo-controlled trial. Lancet Oncol 10: 25-34, 2009.

42. Ali BH, Ziada A and Blunden G: Biological effects of gum arabic: A review of some recent research. Food Chem Toxicol 47: $1-8,2009$.

43. Yu Z, LiHua Y, Qian Y and Yan L: Effect of Lentinus edodes polysaccharide on oxidative stress, immunity activity and oral ulceration of rats stimulated by phenol. Carbohydrate Polymers 75: 115-118, 2009

44. Zong A, Cao $\mathrm{H}$ and Wang F: Anticancer polysaccharides from natural resources: A review of recent research. Carbohydr Polym 90: 1395-1410, 2012.

45. Ajith TA and Janardhanan KK: Cytotoxic and antitumor activities of a polypore macrofungus, Phellinus rimosus (Berk) Pilat. J Ethnopharmacol 84: 157-162, 2003.

46. Santos-Neves JC, Pereira MI, Carbonero ER, Gracher AHP, Alquini G, Gorin PAJ, Sassaki GL and Iacomini M: A novel branched $\alpha \beta$-glucan isolated from the basidiocarps of the edible mushroom Pleurotus florida. Carbohydrate Polymers 73 . 309-314, 2008

47. Wong SM, Wong KK, Chiu LCM and Cheung PCK: Non-starch polysaccharides from different developmental stages of Pleurotus tuber-regium inhibited the growth of human acute promyelocytic leukemia HL-60 cells by cell-cycle arrest and/or apoptotic induction. Carbohydrate Polymers 68: 206-217, 2007.

48. Zhang M, Cheung PCK, Chiu LCM, Wong EYL and Ooi VEC: Cell-cycle arrest and apoptosis induction in human breast carcinoma MCF-7 cells by carboxymethylated $\beta$-glucan from the mushroom sclerotia of Pleurotus tuber-regium. Carbohydrate Polymers 66: 455-462, 2006.

49. Lavi I, Nimri L, Levinson D, Peri I, Hadar Y and Schwartz B: Glucans from the edible mushroom Pleurotus pulmonarius inhibit colitis-associated colon carcinogenesis in mice. J Gastroenterol 47: 504-518, 2012 
50. Maehara Y, Tsujitani S, Saeki H, Oki E, Yoshinaga K, Emi Y, Morita M, Kohnoe S, Kakeji Y, Yano T, et al: Biological mechanism and clinical effect of protein-bound polysaccharide $\mathrm{K}$ $(\operatorname{KRESTIN}(\circledR))$ : Review of development and future perspectives. Surg Today 42: 8-28, 2012

51. Zeng Q, Zhou F, Lei L, Chen J, Lu J, Zhou J, Cao K, Gao L, Xia F, Ding S, et al: Ganoderma lucidum polysaccharides protect fibroblasts against UVB-induced photoaging. Mol Med Rep, 2016.

52. Hu X, Zhang R, Xie Y, Wang $\mathrm{H}$ and Ge M: The protective effects of polysaccharides from Agaricus blazei Murill against cadmium-induced oxidant stress and inflammatory damage in chicken livers. Biol Trace Elem Res, 2016.

53. Li S, Gao A, Dong S, Chen Y, Sun S, LeiZ andZhang Z: Purification, antitumor and immunomodulatory activity of polysaccharides from soybean residue fermented with Morchella esculenta. Int J Biol Macromo 96: 26-34, 2016.

54. Lv Y, Yang X, Zhao Y, Ruan Y, Yang Y and Wang Z: Separation and quantification of component monosaccharides of the tea polysaccharides from Gynostemma pentaphyllum by HPLC with indirect UV detection. Food Chemistry 112: 742-746, 2009.

55. Lu X, Zhao Y, Sun Y, Yang S and Yang X: Characterisation of polysaccharides from green tea of Huangshan Maofeng with antioxidant and hepatoprotective effects. Food Chem 141: 3415-3423, 2013

56. Yang X, Zhao Y, Yang Y and Ruan Y: Isolation and characterization of immunostimulatory polysaccharide from an herb tea, Gynostemma pentaphyllum Makino. J Agric Food Chem 56: 6905-6909, 2008.

57. Chen J, Yong Y, Xing M, Gu Y, Zhang Z, Zhang S and Lu L: Characterization of polysaccharides with marked inhibitory effect on lipid accumulation in Pleurotus eryngii. Carbohydr Polym 97: 604-613, 2013

58. Zhang Y, Dai L, Kong X and Chen L: Characterization and in vitro antioxidant activities of polysaccharides from Pleurotus ostreatus. Int J Biol Macromol 51: 259-265, 2012.

59. Li X, Zhang $\mathrm{H}$ and $\mathrm{Xu} \mathrm{H}$ : Analysis of chemical components of shiitake polysaccharides and its anti-fatigue effect under vibration. Int J Biol Macromol 45: 377-380, 2009.

60. Wang M, Wang H, Tang Y, Kang D, Gao Y, Ke M, Dou J, Xi T and Zhou C: Effective inhibition of a Strongylocentrotus nudus eggs polysaccharide against hepatocellular carcinoma is mediated via immunoregulation in vivo. Immunol Lett 141: 74-82, 2011.

61. Zhang CX and Huang KX: Mechanism of apoptosis induced by a polysaccharide, from the loach Misgurnus anguillicaudatus (MAP) in human hepatocellular carcinoma cells. Toxicol Appl Pharmacol 210: 236-245, 2006

62. Isoda N, Eguchi Y, Nukaya H, Hosho K, Suga Y, Suga T, Nakazawa $S$ and Sugano K: Clinical efficacy of superfine dispersed lentinan ( $\beta$-1,3-glucan) in patients with hepatocellular carcinoma. Hepatogastroenterology 56: 437-441, 2009.

63. Ruan WJ, Lai MD and Zhou JG: Anticancer effects of Chinese herbal medicine, science or myth? J Zhejiang Univ Sci B 7: 1006-1014, 2006.

64. Konkimalla VB and Efferth T: Evidence-based Chinese medicine for cancer therapy. J Ethnopharmacol 116: 207-210, 2008.

65. Niwa Y, Matsuura H, Murakami M, Sato J, Hirai K and Sumi H: Evidence that naturopathic therapy including Cordyceps sinensis prolongs survival of patients with hepatocellular carcinoma. Integr Cancer Ther 12: 50-68, 2013

66. Wang CR, Ng TB, Li L, Fang JC, Jiang Y, Wen TY, Qiao WT, Li N and Liu F: Isolation of a polysaccharide with antiproliferative, hypoglycemic, antioxidant and HIV-1 reverse transcriptase inhibitory activities from the fruiting bodies of the abalone mushroom Pleurotus abalonus. J Pharm Pharmacol 63: 825-832, 2011.

67. Xu WW, Li B, Lai ET, Chen L, Huang JJ, Cheung AL and Cheung PC: Water extract from Pleurotus pulmonarius with antioxidant activity exerts in vivo chemoprophylaxis and chemosensitization for liver cancer. Nutr Cancer 66: 989-998, 2014.

68. Xu W, Huang JJ and Cheung PC: Extract of Pleurotus pulmonarius suppresses liver cancer development and progression through inhibition of VEGF-induced PI3K/AKT signaling pathway. PLoS One 7: e34406, 2012.

69. Xu R, Ye H, Sun Y, Tu Y and Zeng X: Preparation, preliminary characterization, antioxidant, hepatoprotective and antitumor activities of polysaccharides from the flower of tea plant (Camellia sinensis). Food Chem Toxicol 50: 2473-2480, 2012.

70. Chen B, Zhou W, Ning M, Wang Z, Zou L, Zhang H and Wang Q: Evaluation of antitumour activity of tea carbohydrate polymers in hepatocellular carcinoma animals. Int J Biol Macromol 50: 1103-1108, 2012.
71. Liang G, Tang A, Lin X, Li L, Zhang S, Huang Z, Tang H and Li QQ: Green tea catechins augment the antitumor activity of doxorubicin in an in vivo mouse model for chemoresistant liver cancer. Int J Oncol 37: 111-123, 2010.

72. He X, Li X, Liu B, Xu L, Zhao H and Lu A: Down-regulation of Treg cells and up-regulation of TH1/TH2 cytokine ratio were induced by polysaccharide from Radix Glycyrrhizae in H22 hepatocarcinoma bearing mice. Molecules 16: 8343-8352, 2011.

73. Wu S, Liu B, Zhang Q, Liu J, Zhou W, Wang C, Li M, Bao S and Zhu R: Dihydromyricetin reduced Bcl-2 expression via $\mathrm{p} 53$ in human hepatoma HepG2 cells. PLoS One 8: e76886, 2013.

74. Liu J, Shu Y, Zhang Q, Liu B, Xia J, Qiu M, Miao H, Li M and Zhu R: Dihydromyricetin induces apoptosis and inhibits proliferation in hepatocellular carcinoma cells. Oncol Lett 8: 1645-1651, 2014.

75. Zhang Q, Liu J, Liu B, Xia J, Chen N, Chen X, Cao Y, Zhang C, Lu C, Li M and Zhu R: Dihydromyricetin promotes hepatocellular carcinoma regression via a p53 activation-dependent mechanism. Sci Rep 4, 4628: 2014.

76. Jiang L, Zhang Q, Ren H, Ma S, Lu C, Liu B, Liu J, Liang J, Li M and Zhu R: Dihydromyricetin enhances the chemo-sensitivity of nedaplatin via regulation of the $\mathrm{p} 53 / \mathrm{Bcl}-2$ pathway in hepatocellular carcinoma cells. PLoS One 10: e0124994, 2015.

77. Liu B, Zhou W, Chen X, Xu F, Chen Y, Liu J, Zhang Q, Bao S, Chen N, Li M and Zhu R: Dihydromyricetin induces mouse hepatoma Hepal-6 cell apoptosis via the transforming growth factor- $\beta$ pathway. Mol Med Rep 11: 1609-1614, 2015.

78. Lever R and Page C: Novel drug development opportunities for heparin. Nat Rev Drug Discov 1: 140-148, 2002.

79. Presta M, Leali D, Stabile H, Ronca R, Camozzi M, Coco L, Moroni E, Liekens S and Rusnati M: Heparin derivatives as angiogenesis inhibitors. Curr Pharm Des 9: 553-566, 2003.

80. Ferro V, Dredge K, Liu L, Hammond E, Bytheway I, Li C, Johnstone K, Karoli T, Davis K, Copeman E and Gautam A: PI-88 and novel heparan sulfate mimetics inhibit angiogenesis. Semin Thromb Hemost 33: 557-568, 2007.

81. Dredge K, Hammond E, Davis K, Li CP, Liu L, Johnstone K, Handley P, Wimmer N, Gonda TJ, Gautam A, et al: The PG500 series: Novel heparan sulfate mimetics as potent angiogenesis and heparanase inhibitors for cancer therapy. Invest New Drugs 28: 276-283, 2010

82. Budhu A and Wang XW: The role of cytokines in hepatocellular carcinoma. J Leukoc Biol 80: 1197-1213, 2006.

83. Nakamoto Y, Guidotti LG, Kuhlen CV, Fowler P and Chisari FV: Immune pathogenesis of hepatocellular carcinoma. J Exp Med 188: 341-350, 1998.

84. Chuma M, Hige S, Nakanishi M, Ogawa K, Natsuizaka M, Yamamoto Y and Asaka M: 8-Hydroxy-2'-deoxy-guanosine is a risk factor for development of hepatocellular carcinoma in patients with chronic hepatitis $\mathrm{C}$ virus infection. J Gastroenterol Hepatol 23: 1431-1436, 2008.

85. Tanaka H, Fujita N, Sugimoto R, Urawa N, Horiike S, Kobayashi Y, Iwasa M, Ma N, Kawanishi S, Watanabe S, et al: Hepatic oxidative DNA damage is associated with increased risk for hepatocellular carcinoma in chronic hepatitis $\mathrm{C}$. Br J Cancer 98: 580-586, 2008.

86. Marra M, Sordelli IM, Lombardi A, Lamberti M, Tarantino L, Giudice A, Stiuso P, Abbruzzese A, Sperlongano R, Accardo M, et al: Molecular targets and oxidative stress biomarkers in hepatocellular carcinoma: An overview. J Transl Med 9: 171, 2011

87. Bishayee A: The role of inflammation and liver cancer. Adv Exp Med Biol 816: 401-435, 2014.

88. Klampfer L: Cytokines, inflammation and colon cancer. Curr Cancer Drug Targets 11: 451-464, 2011

89. Chang HL, Lei LS, Yu CL, Zhu ZG, Chen NN and Wu SG: Effect of Flammulina velutipes polysaccharides on production of cytokines by murine immunocytes and serum levels of cytokines in tumor-bearing mice. Zhong Yao Cai 32: 561-563, 2009. (In Chinese)

90. Chen G, Xu J, Miao X, Huan Y, Liu X, Ju Y and Han X: Characterization and antitumor activities of the water-soluble polysaccharide from Rhizoma Arisaematis. Carbohydr Polym 90: 67-72, 2012.

91. Wang LJ, Bai L, Su D, Zhang T and Mao ZY: Proinflammatory conditions promote hepatocellular carcinoma onset and progression via activation of Wnt and EGFR signaling pathways. Mol Cell Biochem 381: 173-181, 2013.

92. Cassetta L, Cassol E and Poli G: Macrophage polarization in health and disease. ScientificWorldJournal 11: 2391-2402, 2011. 
93. Yang J, Li X, Xue Y, Wang N and Liu W: Anti-hepatoma activity and mechanism of corn silk polysaccharides in $\mathrm{H} 22$ tumor-bearing mice. Int J Biol Macromol 64: 276-280, 2014.

94. Liu L, Jia J, Zeng G, Zhao Y, Qi X, He C, Guo W, Fan D, Han G and Li Z: Studies on immunoregulatory and anti-tumor activities of a polysaccharide from Salvia miltiorrhiza Bunge. Carbohydr Polym 92: 479-483, 2013.

95. Tian QE, Li HD, Yan M, Cai HL, Tan QY and Zhang WY: Astragalus polysaccharides can regulate cytokine and $\mathrm{P}$-glycoprotein expression in $\mathrm{H} 22$ tumor-bearing mice. World J Gastroenterol 18: 7079-7086, 2012.

96. Schild L, Chen BH, Makarov P, Kattengell K, Heinitz K and Keilhoff G: Selective induction of apoptosis in glioma tumour cells by a Gynostemma pentaphyllum extract. Phytomedicine 17 589-597, 2010

97. Chen J, Chen J, Wang X and Liu C: Anti-tumour effects of polysaccharides isolated from Artemisia annua $\mathrm{L}$ by inducing cell apoptosis and immunomodulatory anti-hepatoma effects of polysaccharides. Afr J Tradit Complement Altern Med 11: $15-22,2013$.

98. Liang M, Li S, Shen B, Cai JP, Li C, Wang ZY, Li XG, Gao J, Huang HY, Zhang XY and Li JY: Anti-hepatocarcinoma effects of Aconitum coreanum polysaccharides. Carbohydrate Polymers 88: 973-976, 2012.

99. Zhang W, Li J, Qiu S, Chen J and Zheng Y: Effects of the exopolysaccharide fraction (EPSF) from a cultivated Cordyceps sinensis on immunocytes of $\mathrm{H} 22$ tumor bearing mice. Fitoterapia 79: $168-173,2008$.

100. Byeon SE, Lee J, Lee E, Lee SY, Hong EK, Kim YE and Cho JY: Functional activation of macrophages, monocytes and splenic lymphocytes by polysaccharide fraction from Tricholoma matsutake. Arch Pharm Res 32: 1565-1572, 2009.
101. Kim JY, Byeon SE, Lee YG, Lee JY, Park J, Hong EK and Cho JY: Immunostimulatory activities of polysaccharides from liquid culture of pine-mushroom Tricholoma matsutake. J Microbiol Biotechnol 18: 95-103, 2008.

102. Ren M, Ye L, Hao X, Ren Z, Ren S, Xu K and Li J: Polysaccharides from Tricholoma matsutake and Lentinus edodes enhance 5-fluorouracil-mediated H22 cell growth inhibition. J Tradit Chin Med 34: 309-316, 2014

103. Kim DH and Rossi JJ: Strategies for silencing human disease using RNA interference. Nat Rev Genet 8: 173-184, 2007.

104. Takeshita F and Ochiya T: Therapeutic potential of RNA interference against cancer. Cancer Sci 97: 689-696, 2006.

105. Whitehead KA, Langer R and Anderson DG: Knocking down barriers: Advances in siRNA delivery. Nat Rev Drug Discov 8: 129-138, 2009.

106. Huang Z, Dong L, Chen J, Gao F, Zhang Z, Chen J and Zhang J: Low-molecular weight chitosan/vascular endothelial growth factor short hairpin RNA for the treatment of hepatocellular carcinoma. Life Sci 91: 1207-1215, 2012.

107. Xu Y, Wen Z and Xu Z: Chitosan nanoparticles inhibit the growth of human hepatocellular carcinoma xenografts through an antiangiogenic mechanism. Anticancer Res 29: 5103-5109, 2009

108. Han L, Tang C and Yin C: Oral delivery of shRNA and siRNA via multifunctional polymeric nanoparticles for synergistic cancer therapy. Biomaterials 35: 4589-4600, 2014

109. Roy M, Luo YH, Ye M and Liu J: Nonsmall cell lung cancer therapy: Insight into multitargeted small-molecule growth factor receptor inhibitors. Biomed Res Int 2013: 964743, 2013

110. Wang E, Chen X, Wang K, Wang J, Chen D, Geng Y, Lai W and Wei X: Plant polysaccharides used as immunostimulants enhance innate immune response and disease resistance against Aeromonas hydrophila infection in fish. Fish Shellfish Immunol 59: 196-202, 2016. 